\title{
Pediatric Heart Rate Variability Normative Values Related to Average Heart Rate and Age in a Developing Country
}

\author{
Çelik Serkan Fazlı1 ${ }^{1 *}$ and Elif Çelik ${ }^{2}$ \\ ${ }^{1}$ Department of Pediatrics, Division of Pediatric Cardiology, Adnan Menderes University Hospital, Aydın, Turkey \\ ${ }^{2}$ Department of Pediatrics, Adnan Menderes University Hospital, Aydın, Turkey
}

*Corresponding author: Çelik Serkan Fazlı, Department of Pediatrics, Division of

Pediatric Cardiology, Adnan Menderes University Hospital, Aydın, Turkey.

Received Date: September 05, 2019

Published Date: September 11, 2019

\begin{abstract}
Abbreviations: HRV: Heart rate variability; ANS: Autonomic nervous system changes; HF: High frequency; LF: Low frequency: MRR: Mean RRI; PNN50: Percentage of consecutive normal RRI differing more than $50 \mathrm{~ms}$; RMSSD: Root mean square of successive differences; SDNN: Standard deviation of normal-to-normal RR intervals; SD1: Standard deviation measuring the dispersion of points in the Poincare plot of RR intervals across the identity line; SD2: Standard deviation measuring the dispersion of points in the Poincare plots of RR intervals along the identity line; CL: The contribution of the long-term HRV to the total HRV that is a percentage of the long-term variance (SD22) of RR intervals to doubled total variance of RR intervals (SDNN2)
\end{abstract}

\section{Introduction}

Cardiac autonomic function can evaluate with Heart Rate Variability (HRV) which is a noninvasive diagnostic method using mathematical algorithms [1]. In the past, many studies have been used to obtain information about the variability of the heart rate [25]. Although there are too many reference values for HRV parameters among adults in each sex and age group [6], there is not enough pediatric study in the literature [7-10]. The recent pediatric studies notice that autonomic nervous system (ANS) changes are associated with behavioral problems and overweight in children. Moreover, De Bock et al. suggested that in normal children, ANS activity changes over time and these changes lead to a decrease in vagal activity and predominance in sympathetic activity [11]. Eyre et al claimed that there may be a genetic difference making different ethnic groups more vulnerable to abnormal cardiovascular risk profiles [12]. These novel observations may have important consequences for our understanding of the high cardiovascular risk profile of which may emerge in childhood and develop in adulthood. Unfortunately, all of pediatric HRV studies were from development coutries, and there is no study to assess normative values of cardiac autonomic function in Turkey. The first aim of the present study was to identify normative values and the main factors of HRV in healthy children in a developing country. The secondary aim of the present study was to analyze the relationship between gender and age in healthy children and their effects on HRV with 24-hour ECG Holter records.

\section{Materials and Methods}

\section{Study population}

This study covers the period between January 2017 and November 2017. The study sample consisted of 120 non-athlete healthy children who included in this study were selected as the control group of 2 different HRV studies previously. The reasons for visits of these healthy children were cardiac palpitations, innocent murmurs, and chest pain. Anthropometric and blood pressure measurements of the cases were performed. Blood samples were obtained from each patient for the analysis of serum folic acid, Hcy, B12 levels, ferritin, hemogram, blood urea nitrogen, creatinine, cholesterol, triglyceride, high-density lipoprotein, low-density lipoprotein, aspartate aminotransferase, alanine aminotransferase, and lactate dehydrogenase levels were measured in all groups. Exclusion criteria were obesity, anemia, arrhythmias medication, psychosomatic and psychiatric disease or acute and chronic disease. The study protocol conforms to the Helsinki Declaration (2013) and was approved by the local ethical committee of University. Each patient gave informed consent. The group was divided into 
two subgroups according to age: Group (1); 7 -12 years old ,and Group (2); 12 -18 years old.

\section{4-h ECG holter recording}

Twenty-four hours ambulatory electrocardiogram recordings were taken with Biomedical Instruments Holter recorder. The recordings with three-channel ECG were reviewed to confirm $24 \mathrm{~h}$ of clear recording which included the data from the whole night, and the classification of beats were manually checked and corrected.

\section{Heart Rate and heart rate variability}

Heart Rate Variability parameters of time and long-term frequency domains were calculated using the software present in the system ECGLab Holter Analysis System Version 1.0 (Biomedical Instruments, China). In time-domain analysis; all the RR intervals, the percentage of the beats with consecutive RR interval difference of $>50 \mathrm{~ms}$ ( $\mathrm{pNN} 50$ ), and the square root of the mean of the squared differences of two consecutive RR intervals (rMSSD) were calculated. We quantified following HRV parameters: SDNN-standard deviation of normal-to-normal RR intervals as a measure of total HRV, SD1; Standard deviation measuring the dispersion of points in the Poincare plot of RR intervals across the identity line, SD2; Standard deviation measuring the dispersion of points in the Poincare plots of RR intervals along the identity line, CL; the contribution of the long-term HRV to the total HRV that is a percentage of the long-term variance (SD22) of RR intervals to doubled total variance of RR intervals (SDNN2) [6]. In frequencydomain analysis; high-frequency power (HF) (the area under the spectral curve from 0.15 to $0.40 \mathrm{~Hz}$ ), low-frequency power (LF) (the area under the spectral curve from 0.04 to $0.15 \mathrm{~Hz}$ ), and the ratio LF/HF were calculated. In frequency-domain analysis, LF was accepted as equivalent to the sympathetic plus parasympathetic components of autonomic function, the parasympathetic component of autonomic function was calculated with HF, and LF/ HF was accepted as the sympathetic- parasympathetic balance of the autonomic function [13].

\section{HRV correction}

In the present study, for HRV correction, we used the same method which Sacha et al. explained before (14). If HRV parameters were negatively disclosed with HR, we divided HRV parameters to the mean RR interval (mRR) in order to become HR independent, in contrast to the HRV parameters were positively disclosed with HR, we multiplied with mRR.

\section{Statistical analysis}

The numerical parameters were reported as mean \pm standard deviation. We used Student's t-test to compare the mean values of normal distribution variables. If data distribution was not Gaussian, the Shapiro-Wilk test used and so these results were showed as mean, Standard deviation (SD), and median. We used the non-parametric Mann-Whitney test to compare HRV parameters between boys and girls, and the Man-Whitney test was used to determine the issue of interaction between sex and age in the sujects. All children were divided into two age groups. For comparison between age groups, the Kruskal-Wallis test was used. Also, for the analysis of the relationship between HRV parameters and age, we applied the nonparametric Spearman correlation. Statistical analysis was carried out using SPSS 22 .0 (SPSS Inc.,Chicago, IL, USA) was used for data management and analysis. $\mathrm{P}<0.05$ was considered significant.

\section{Result}

The $24 \mathrm{~h}$ ECG recordings were performed in a total of 129 healthy subjects. Five of subjects with technically inadequate ECG recordings, four subjects had frequently supra ventricular extra-systoles and they were excluded from the study (Figure 1). Finally, 62 girls and 58 boys ( mean age $12.9 \pm 4.3$ years, age range 7-18 years) included in the present study (Table 1). The Shapiro-Wilk test determined no skewed distribution in examined variables. Total ECG recordings duration was $23.21 \pm 1.2$ and there was no difference between groups ( $p>0.05)$. The results of HRV measurements are shown in (Table 2). The values of ULF, LF, SDNN, LF/HF significantly increased, but VLF, HF and rMSSD did not change significantly with age. The alteration of the time of corrected QT (QTc) was not associated with the age group. However, time of QRS and mRR significantly increased with age. The HRV parameters were not influenced by gender (Table 3). In accordance with Spearman correlation test, there were significant, positive and strong correlations among the age and SDNN, LF, ULF, LF/HF and the duration of mean RR interval (Table 4).

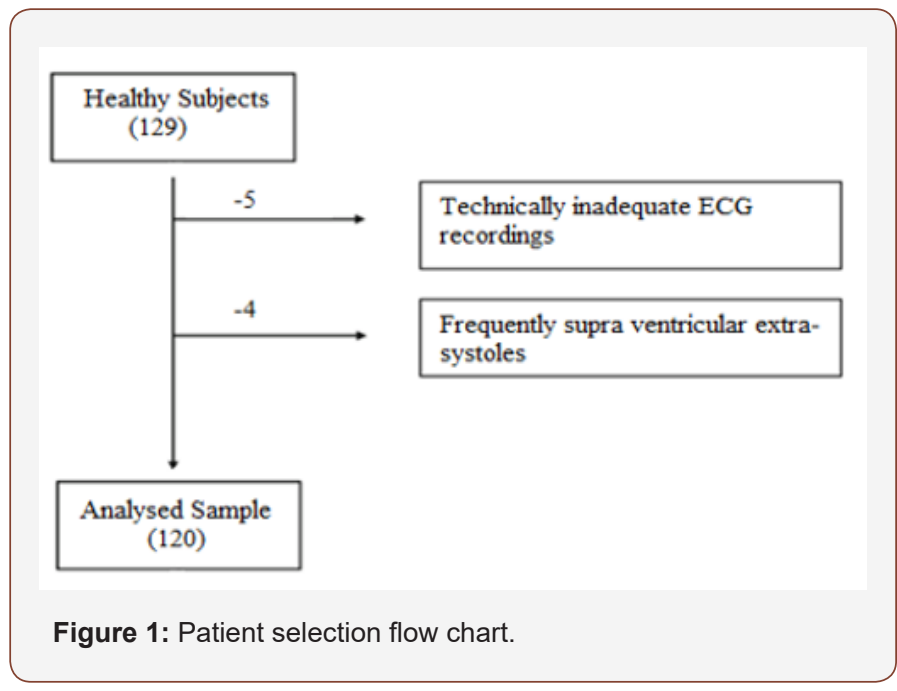

Table 1: Clinical characteristics of participants.

\begin{tabular}{|c|c|c|c|c|}
\hline & Boys & Girls & p & Total \\
\hline $\mathrm{n}$ & 58 & 62 & 0.14 & 120 \\
\hline Age (y) & $12.8 \pm 3.2$ & $13.1 \pm 3.3$ & 0.34 & $12.9 \pm 4.3$ \\
\hline $\begin{array}{c}\text { BMI (kg/ } \\
\text { m2) }\end{array}$ & $21.6 \pm 0.5$ & $22.1 \pm 0.7$ & 42 & $21.9 \pm 0.6$ \\
\hline $\begin{array}{c}\text { SBP } \\
(\mathrm{mmHg})\end{array}$ & $104.6 \pm 8.6$ & $102.7 \pm 7.5$ & 0.44 & $103.4 \pm 7.9$ \\
\hline $\begin{array}{c}\text { DBP } \\
(\mathrm{mmHg})\end{array}$ & $66.4 \pm 7.8$ & $61.8 \pm 4.5$ & 0.12 & $64.2 \pm 6.1$ \\
\hline $\begin{array}{c}\text { Heart rate } \\
\text { (b/min) }\end{array}$ & $79.6 \pm 14.4$ & $81.4 \pm 16.3$ & 0.25 & $80.6 \pm 15.8$ \\
\hline \multicolumn{2}{|c|}{ Values are } & & &
\end{tabular}

Values are means \pm SD. BMI; body mass index, DBP; diastolic blood pressure, $\mathrm{SBP}$; systolic blood pressure, $\mathrm{b} / \mathrm{min}$; beats per minute. $\mathrm{p}<$ 0.05 . 
Table 2: The comparison of parameters of the HRV of the current sample categorized by age groups. was analysed with the non-parametric KruskalWallis test.

\begin{tabular}{|c|c|c|c|c|c|c|c|c|c|c|c|}
\hline & \multicolumn{5}{|c|}{ Group $1 \mathrm{~N}=60$} & \multicolumn{5}{|c|}{ Group $2 \mathrm{~N}=60$} & \multirow[t]{2}{*}{$\mathbf{p}$} \\
\hline & Mean & SD & Median & 25th p. & 75th p. & Mean. & SD & Median & 25th p. & 75th p. & \\
\hline $\mathrm{PQ}[\mathrm{ms}]$ & 130.1 & 19.4 & 128.06 & 111 & 141.2 & 132.6 & 15.1 & 129.9 & 118 & 141.1 & 0.12 \\
\hline QRS [ms] & 86.1 & 10.4 & 84.4 & 80.01 & 91 & 89.4 & 11.4 & 88.1 & 82.1 & 92.9 & 0.04 \\
\hline QTc [ms] & 390.4 & 17.1 & 392.6 & 380 & 404.5 & 391.5 & 22.6 & 405.5 & 389.6 & 417.9 & 0,80 \\
\hline Mean RR (ms) & 686.7 & 68.2 & 700.8 & 607.6 & 736.7 & 750.7 & 75.1 & 785.2 & 684.1 & 856.6 & 0.007 \\
\hline SDNN (ms) & 154.1 & 40.2 & 145.9 & 134.6 & 187.5 & 161.9 & 42.4 & 160.6 & 134.3 & 187.8 & 0.01 \\
\hline RMSSD (ms) & 44 & 35.4 & 38.6 & 31.5 & 66.4 & 46.5 & 36.5 & 38.9 & 41.3 & 68.3 & 0.36 \\
\hline ULF [ms2] & 16.17 & 10.46 & 13.9 & 9.45 & 21.74 & 24.82 & 11.31 & 23.67 & 14.78 & 32.36 & 0.002 \\
\hline VLF [ms2] & 3261.8 & 1801.6 & 2598.9 & 1986.7 & 4287.8 & 4265.1 & 2014.6 & 4191.6 & 2617.8 & 5419.4 & 0.16 \\
\hline LF [ms2] & 1889.5 & 1116 & 1485.8 & 1088.4 & 2384.9 & 2089.8 & 1174.9 & 1885.5 & 1238.8 & 2793.7 & 0.035 \\
\hline $\mathrm{HF}$ [ms2] & 2308.4 & 1958.4 & 1435.9 & 776.8 & 2962.5 & 2212.9 & 1814.1 & 1477.7 & 699.6 & 2185 & 0.62 \\
\hline $\mathrm{LF} / \mathrm{HF}$ & 1.3 & 0.6 & 1.2 & 1 & 1.4 & 1.6 & 0.8 & 1.6 & 1.3 & 2.1 & 0.03 \\
\hline SD1 [ms] & 54.5 & 31.6 & 49.1 & 31.7 & 70.2 & 49.6 & 24.6 & 46.9 & 53.4 & 59.7 & 0.3 \\
\hline $\mathrm{SD} 2[\mathrm{~ms}]$ & 214.6 & 58.7 & 205 & 172.6 & 246.4 & 239.4 & 59.7 & 241.3 & 192.6 & 286.6 & 0.39 \\
\hline CL [\%] & 93.1 & 5.7 & 94.7 & 90.8 & 96.2 & 96.3 & 3.9 & 97.6 & 95.1 & 98.5 & 0.12 \\
\hline
\end{tabular}

Table 3: The comparison of HRV analysis between girls and boys was analysed with the Mann-Whitney test.

\begin{tabular}{|c|c|c|c|c|c|c|c|c|c|c|c|}
\hline & \multicolumn{5}{|c|}{ GIRLS $\quad N=62$} & \multicolumn{5}{|c|}{ BOYS $N=58$} & \multirow[t]{2}{*}{ p-value } \\
\hline & & & & 25th p. & 75th p. & Mean. & SD & Median & 25th p. & 75th p. & \\
\hline & Mean. & SD & Median & 25th p. & 75th p. & Mean. & SD & Median & 25th p. & 75th p. & \\
\hline $\mathrm{PQ}[\mathrm{ms}]$ & 126.4 & 15.1 & 138.6 & 118.2 & 141.7 & 127.1 & 15.8 & 139.1 & 124.8 & 143.6 & 0.64 \\
\hline QRS [ms] & 84.6 & 9.6 & 79.01 & 78,2 & 89.7 & 88.5 & 9.7 & 81.64 & 80.6 & 92.6 & 0.04 \\
\hline QTc [ms] & 390.8 & 17.5 & 389 & 377.3 & 401.5 & 381.1 & 19.7 & 391.06 & 372.6 & 399.1 & 0.87 \\
\hline Mean RR (ms) & 740.1 & 74.1 & 741.5 & 696.3 & 763.7 & 734.4 & 87.2 & 740.4 & 699.2 & 789.4 & 0.91 \\
\hline SDNN (ms) & 157.6 & 45.4 & 160.8 & 129.4 & 188.8 & 156.4 & 44.9 & 159.6 & 130.4 & 192.8 & 0.2 \\
\hline RMSSD (ms) & 43.1 & 34.6 & 37.8 & 30.6 & 64.2 & 45.5 & 38.8 & 40.1 & 42.4 & 69.5 & 0.44 \\
\hline ULF [ms2] & 21.64 & 12.44 & 19.84 & 13,56 & 31.4 & 20.95 & 10.94 & 18,193 & 10.01 & 30.8 & 0.64 \\
\hline VLF [ms2] & 3421 & 1710 & 32.45 & 1848.4 & 3614.5 & 3628.5 & 1941.4 & 3340 & 2061.6 & 3852.8 & 0.85 \\
\hline LF [ms2] & 1925.6 & 1164.9 & 1765.5 & 1072.6 & 2399 & 2016.1 & 1185.8 & 1874.4 & 1174.4 & 30546 & 0.64 \\
\hline $\mathrm{HF}$ [ms2] & 1752.6 & 1988.1 & 1501.4 & 852.4 & 1934.4 & 1874.6 & 1685.8 & 1621.7 & 791.6 & 2065.4 & 0.38 \\
\hline $\mathrm{LF} / \mathrm{HF}$ & 1.6 & 0.8 & 1.4 & 1.2 & 1.9 & 1.7 & 0.7 & 1.3 & 1 & 2.1 & 0.24 \\
\hline $\mathrm{SD} 1[\mathrm{~ms}]$ & 44.5 & 22.5 & 40.8 & 22.5 & 66.4 & 47.8 & 21.3 & 44.4 & 22.4 & 68.4 & 0.46 \\
\hline $\mathrm{SD} 2[\mathrm{~ms}]$ & 211.5 & 50.5 & 207.3 & 159.7 & 256.6 & 212.1 & 51.56 & 201.6 & 162.2 & 260.5 & 0.51 \\
\hline CL [\%] & 94.5 & 4.2 & 92.5 & 90.2 & 99.6 & 93.8 & 4.1 & 91.5 & 92.6 & 98.4 & 0.15 \\
\hline
\end{tabular}

Table 4: The results of Spearman correlation between HRV parameters and the age of subjects.

\begin{tabular}{|c|c|c|}
\hline & Rho & P-value \\
\hline Mean RR & 0.36 & $<0.001$ \\
\hline SDNN & 0.54 & 0.008 \\
\hline ULF & 0.421 & $<0.001$ \\
\hline VLF & 0.37 & 0.17 \\
\hline LF & 0.25 & 0.028 \\
\hline LF/HF & 0.16 & 0.001 \\
\hline
\end{tabular}

\section{Discussion}

Although there are considerable studies on HRV in children in development countries, there is no study about normative values for HRV parameters in healthy children in a developing country.
The first aim of the present study was to define normative values for HRV parameters in healthy children in Turkey. Secondly, HRV reflects developmental ANS activity. In order to follow developmental alterations during life time period, we need to establish normal values for HRV parameters. Recently, Lower HRV parameters were observed in children with Fontan circulation [14], in children with congenital heart diseases [15], and in children with type 1 diabetes [16] compared with healthy controls. Dias and et al. revealed that children with the attention deficit hyperactivity disorder had higher HRV parameters showing the parasympathetic activity [17]. The ethnic differences of HRV and normal HRV values for other populations in children were shown previously. Reed et al claimed that Caucasian and Asian children had distinct frequency domain components of HRV in Canada [18]. Eyre et al confirmed 
that White European children have higher HRV values than South Asian children and this increase is presumably not related to physical activity or aerobic fitness [12].

In our study, we examined significant correlations between most time domain of HRV parameters and age. HRV parameters increased significantly with age, except HF and rMSSD. Our results are in coherent with previously reported studies $[19,20]$. Cysarz et al. showed that HF did not change in children older than 13 years old, and then lightly declined, but generally HF was not correlated with age. Our analysis displayed that age and HR are the strongest predictors for most of standard HRV parameters. This effect has been shown in some studies in children [20-22]. İn a recent study, healthy children and young adults using Holter ECG recordings were examined and claimed that total power (TP) was not changed with age and the awareness or sleep state, HF only changed during quiet sleep, LF and LF/HF both changed significantly with age during silent and active sleep period [19]. Unlike most of recent studies, we used the HRV correction analysis and 24-h Holter ECG recordings. The present study's results were similar with the study of Gasior et al [23]. However, Gasior et al used only five-minute electrocardiograms, this is a methodological problem for their study [13]. Also, Jarrin et al presented the calibrated normative values for HR in children [24]. Nevertheless, age of their study group was very narrow (9-11 years). In the present study, we found that sex had no impact on HRV parameters. The impact of sex on HRV is controversial. Silvetti et al. studied on time domain HRV parameters in healthy children and found none of HRV parameters were related to gender [25]. Similarly, Goto et al. found no significant differences by genders in healthy children [20]. Inversely, Galeev et al. investigated 5,400 children in the age 6-16, and finally found that SDNN was significantly more increased in boys than in girls for between 12 and 14 years old children. Total power was significantly lower in girls between 11 and 15 years old, VLF was lower in girls between 13 and 15 years old but higher for 12 years old. The effect of sex on LF and HF were presented for children, morever the LF/HF was smaller in girls than in boys in the age 12-15 [26]. Gasior et al. recorded a resting ECG in 331 children and boys presented significantly lower HR with higher HRV values [23]. However, when they analyse with regression analysis adjusted for gender, HRV parameters did not relate to sex but they relate to heart rate. Similar to our study, Bobkowski et al. used plots and Periodograms and found that HRV parameters were not affected from gender. Nevertheless, most of HRV parameters increased with age in healthy children [27]. To the best of our knowledge, this study is the first report on HRV in healthy children in Turkey, and for this reason other authors in developing countries may use these results as reference values.

\section{Limitations of the study}

In the present study, the number of patients is relatively small. We used HRV which is an indirect method to measure ANS, but HRV is recommended for measuring ANS in specific guidelines. Although athlete healthy children were excluded from the study, we were unable to match children on physical activity.

\section{Conclusion}

This study demonstrates HRV normative values for children with considering differences, especially related to average HR and age in Turkey.

\section{Acknowledgement}

The retrospective datas of healthy children in this study were included from two previous studies and this study was approved by the local ethical committee of University.

\section{Conflict of Interest}

No conflict of interest.

\section{References}

1. Sassi R, Cerutti S, Lombardi F, Malik M, Huikuri HV, et al. (2015) Advances in heart rate variability signal analysis: joint position statement by the e-Cardiology ESC Working Group and the European Heart Rhythm Association co-endorsed by the Asia Pacific Heart Rhythm Society. Ep Europace 17: 1341-1353.

2. Bigger JT, Fleiss JL, Rolnitzky LM, Steinman RC (1993) Frequency domain measures of heart period variability to assess risk late after myocardial infarction. Journal of the American College of Cardiology 21: 729-736.

3. Patel VN, Pierce BR, Bodapati RK, Brown DL, Ives DG, et al. (2017) Association of holter-derived heart rate variability parameters with the development of congestive heart failure in the cardiovascular health study. JACC: Heart Failure 5(6): 423-431.

4. Akinci A, Çeliker A, Baykal E, Teziç T (1993) Heart rate variability in diabetic children: sensitivity of the time-and frequency-domain methods. Pediatric Cardiology 14: 140-146.

5. Stein PK, Reddy A (2005) Non-linear heart rate variability and risk stratification in cardiovascular disease. Indian Pacing and Electrophysiology Journal 5(3): 210-220.

6. (1996) Variability HR Standards of measurement, physiological interpretation, and clinical use. Task Force of the European Society of Cardiology and the North American Society of Pacing and Electrophysiology. Circulation 93: 1043-1065.

7. Winsley R, Armstrong N, Bywater K, Fawkner S (2003) Reliability of heart rate variability measures at rest and during light exercise in children. British journal of sports medicine 37: 550-552.

8. Schroeder EB, Whitsel EA, Evans GW, Prineas RJ, Chambless LE, et al. (2004) Repeatability of heart rate variability measures. Journal of electrocardiology 37: 163-172.

9. Bjelakovic B, Ilic S, Dimitrijevic L, Milovanovic B, Kostic G, et al. (2010) Heart rate variability in infants with central coordination disturbance. Early human development 86: 77-81.

10. McNarry MA, Mackintosh KA (2016) Reproducibility of heart rate variability indices in children with cystic fibrosis. PloS one 11: e0151464.

11. De Bock F, Jarczok MN, Hoffmann K, Buchhorn R (2013) Do our children lose vagus activity? Potential time trends of children's autonomic nervous system activity. Int J Cardiol 170: e30-2.

12. Eyre EL, Fisher JP, Smith EC, Wagenmakers AJ, Matyka KA, et al. (2013) Ethnicity and long-term heart rate variability in children. Archives of disease in childhood 98(4): 292-298.

13. Camm A, Malik M, Bigger J, Breithardt G, Cerutti S, et al. (1996) Heart rate variability: standards of measurement, physiological interpretation and clinical use. Task Force of the European Society of Cardiology and the North American Society of Pacing and Electrophysiology. Circulation 93: 1043-65.

14. Dahlqvist JA, Karlsson M, Wiklund U, Hörnsten R, Strömvall-Larsson E, et al. (2012) Heart rate variability in children with Fontan circulation: lateral tunnel and extracardiac conduit. Pediatric cardiology 33: 307315. 
15. Massin M, Von Bernuth G (1998) Clinical and haemodynamic correlates of heart rate variability in children with congenital heart disease European Journal of pediatrics 157: 967-971.

16. Kardelen F, Akçurin G, Ertuğ H, Akcurin S, Bircan I, et al. (2006) Heart rate variability and circadian variations in type 1 diabetes mellitus. Pediatric diabetes 7: 45-50.

17. De Carvalho TD, Wajnsztejn R, De Abreu LC, Vanderlei LCM, Godoy MF, et al. (2014) Analysis of cardiac autonomic modulation of children with attention deficit hyperactivity disorder. Neuropsychiatric disease and treatment 10: 613-618.

18. Reed KE, Warburton DE, Whitney CL, McKay HA (2006) Differences in heart rate variability between Asian and Caucasian children living in the same Canadian community. Applied physiology, nutrition, and metabolism 31: 277-282.

19. Finley JP, Nugent ST (1995) Heart rate variability in infants, children and young adults. Journal of the autonomic nervous system 51: 103-108.

20. Goto M, Nagashima M, Baba R, Nagano Y, Yokota M, et al. (1997) Analysis of heart rate variability demonstrates effects of development on vagal modulation of heart rate in healthy children. The Journal of pediatrics 130: 725-729.

21. Bjelakovic B, Ilic D, Lukic S, Vukomanovic V, Zarko C, et al. (2017) Reproducibility of $24-\mathrm{h}$ heart rate variability in children. Clinical Autonomic Research 27: 273-278.
22. Herzig D, Eser P, Radtke T, Wenger A, Rusterholz T, et al. (2017) Relation of heart rate and its variability during sleep with age, physical activity, and body composition in young children. Frontiers in physiology 8: 109.

23. Gąsior JS, Sacha J, Pawłowski M, Zieliński J, Jeleń PJ, et al. (2018) Normative Values for Heart Rate Variability Parameters in School-Aged Children: Simple Approach Considering Differences in Average Heart Rate. Frontiers in physiology 9: 1495.

24. Jarrin DC, McGrath JJ, Poirier P, Séguin L, Tremblay RE, et al. (2015) Shortterm heart rate variability in a population-based sample of 10-year-old children. Pediatric cardiology 36: 41-48.

25. Silvetti MS, Drago F, Ragonese P (2001) Heart rate variability in healthy children and adolescents is partially related to age and gender. International journal of cardiology 81: 169-174.

26. Galeev A, Igisheva L, Kazin E (2002) Heart rate variability in healthy sixto sixteen-year-old children. Human Physiology 28: 428-432.

27. Bobkowski W, Stefaniak ME, Krauze T, Gendera K, Wykretowicz A, et al. (2017) Measures of heart rate variability in 24-h ECGs depend on age but not gender of healthy children. Frontiers in physiology 8: 311. 